\title{
NONCOMBINATORIAL TRIANGULATIONS AND THE POINCARE CONJECTURE
}

\author{
RALPH TINDELL ${ }^{1}$
}

Kirby and Siebenmann have recently proved that every boundaryless topological $n$-manifold ( $n \geqq 5$ ) having trivial 4-dimensional $Z_{2^{-}}$ cohomology can be given a combinatorial triangulation; they have also given an example of a topological manifold which supports no combinatorial triangulation. It is quite possible, however, that the manifold constructed might be triangulable by a complex which is not a combinatorial manifold. The main purpose of this note is to show that if this could be done in such a way that the open simplexes are locally flat, then either the 3- or the 4-dimensional Poincare conjecture would be false. Let us call such a triangulation locally flat; note that we do not require that the closed simplexes be locally flat. Then our main result is:

Theorem 3. Suppose $n \geqq 5$. Then the 3- and 4-dimensional Poincaré conjectures together are equivalent to the conjecture that every locally flat triangulation of a topological n-manifold is combinatorial.

Let $X \subset Y$ be metric spaces and suppose $z \in X$. A monotone sequence $V_{1} \supset V_{2} \supset \cdots$ of compact neighborhoods of $z$ in $Y$ is said to be a local homotopy sequence for $X$ at $z$ in $Y$ if $\cap V_{i}=\{z\}$ and each inclusion $V_{j} \backslash X$ into $V_{k} \backslash X(k<j)$ is a homotopy equivalence. If such a neighborhood sequence exists, then the homotopy type of $V_{1} \backslash X$ is called the local homotopy type of $X$ at $z$ in $Y$; that the local homotopy type is independent of the choice of defining sequence may be seen as follows: if $U_{i}$ is another such sequence, we may find integers $p, q, r$ such that $U_{r} \subset V_{q} \subset U_{p} \subset V_{1}$. The inclusions form a commutative diagram as follows:

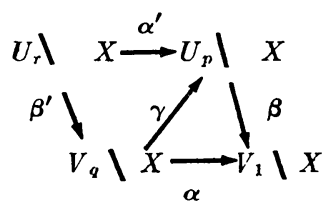

Since $\alpha^{\prime}$ and $\alpha$ are homotopy equivalences, with homotopy inverses

Received by the editors April 14, 1969.

1 The author was partially supported by the National Science Foundation, Grant 8615. 
$\bar{\alpha}^{\prime}$ and $\bar{\alpha}, \beta^{\prime} \bar{\alpha}^{\prime}$ is a right homotopy inverse for $\gamma$ and $\bar{\alpha} \beta$ is a left homotopy inverse for $\gamma$. Hence $\gamma$ is a homotopy equivalence.

There are two instances of importance to us where the local homotopy type is defined: if

Proposition 1. The local homotopy type of $X$ at $z$ in $Y$ is well defined

(i) $X$ is a $k$-manifold in the $n$-manifold $Y$, and $X$ is locally flat at $z$; the local homotopy type is that of the $(n-k-1)$-sphere;

(ii) $z$ is an interior point of a simplex $X$ in a complex $Y$; the local homotopy type is the homotopy type of the link of $X$ in $Y, \operatorname{lk}(X, Y)$.

COROLlaRy 1. If $\sigma^{k}$ is a simplex of a locally flat triangulation of a topological n-manifold, then $\mathrm{lk}(\sigma, K)$ has the homotopy type of $S^{n-k-1}$.

Part (i) of the proposition reduces to the case $X=R^{k}, Y=R^{n}, z$ $=$ origin, and we then define $V_{i}$ to be the ball of radius $1 / i$ about $Z$; $V_{1} \backslash R^{k}$ then deforms to $S^{n-1} \backslash S^{k-1}$, which has the homotopy type of $S^{n-k-1}$.

For part (ii), $V_{1}=\operatorname{st}(X, Y)$ and $V_{i}=\operatorname{st}\left(z, Y^{(i)}\right)$, where $Y^{(i)}$ is an $r$ th derived subdivision of $Y$, with $X$ originally starred at $z$. In this case, $V_{1} \backslash X=\operatorname{st}(X, Y) \backslash X$ deformation retracts to $\mathrm{lk}(X, Y)$.

The corollary then follows from (i) and (ii) by considering the local homotopy type of $\sigma$ at its barycenter.

Theorem 1. The Poincaré conjectures imply that every locally flat triangulation of a topological manifold is combinatorial.

Proof. Assume the Poincaré conjectures, and let $K$ be a triangulation of a topological $n$-manifold. Let $\sigma^{k}$ be a simplex of maximal dimension such that $\operatorname{lk}(\sigma, K)$ is not $\mathrm{PL}$ homeomorphic to $S^{n-k-1}$. If such a simplex exists, then $\mathrm{lk}(\sigma, K)$ must be a combinatorial manifold: if $1 \mathrm{k}(\sigma, K)$ fails to be combinatorial at a vertex $v$, then $\operatorname{lk}\left(v_{\sigma}, K\right)$ $=1 \mathrm{k}(v, \operatorname{lk}(\sigma, K))$ is not a PL-sphere, violating the maximality of $\sigma$. Thus, by Corollary $1, \operatorname{lk}(\sigma, K)$ is a homotopy sphere, and hence, by the Poincaré conjecture, a sphere. In other words, there was no such simplex $\sigma$, so that $K$ is combinatorial.

Lemma 1. If $K$ is combinatorial at $\sigma$, then int $\sigma$ is locally flat.

Proof. The pair $(\operatorname{st}(\sigma, K), \sigma)$ is an unknotted ball pair.

LEMMA 2. If $K$ triangulates a topological $n$-manifold, then $\sigma$ is combinatorial at simplexes of dimension $k \geqq n-3$.

Proof. For each simplex $\sigma$ of $K, \mathrm{lk}(\sigma, K)$ must be a pseudomani- 
fold with the homology of a sphere; if $\operatorname{dim} \sigma \geqq n-3$, then $\operatorname{dim}(\operatorname{lk}(\sigma, K))$ $\leqq 2$, and it is well known that $\operatorname{lk}(\sigma, K)$ must then be a sphere.

Theorem 2. If either the 3-dimensional, or the 4-dimensional Poincaré conjecture is false, then there are locally flat noncombinatorial triangulation of $S^{n}$ for all $n \geqq 5$, and hence for all combinatorial $n$ manifolds, $n \geqq 5$.

Proof. We need only show that there would be a locally flat noncombinatorial triangulation of $S^{5}$, since suspension of a locally flat triangulation gives a locally flat triangulation (recall that "locally flat" means locally flat on open simplexes.) If the 4-dimensional Poincaré conjecture fails, the suspension of a fake 4-sphere is a topological 5-sphere (see, for example, [2]), yielding a noncombinatorial triangulation of $S^{5}$. Since vertices are automatically locally flat, and since such a triangulation would be combinatorial at all other simplexes, it would be a locally flat triangulation. If the 3 -dimensional Poincaré conjecture were false, there would be a fake 3 -sphere $M^{3}$ whose double suspension $S^{1} * M^{3}$ is a topological 5-sphere [2]. If $K$ triangulates $M^{3}$, and $L$ triangulates $S^{1}$, then the join $L * K$ would be a noncombinatorial triangulation of $S^{5} \mathrm{~L} * K$ would be combinatorial at all simplexes except those belonging to $L$. Let $\sigma^{1}$ be an arbitrary simplex of $L$, and let $x$ be an interior point of $\sigma^{1}$. Then the local homotopy type of $S^{1}$ at $x$ in $S^{5}=S^{1} * M^{3}$ is just the homotopy type of $M^{3}$, which is simply connected. As a consequence, the circle $S^{1}$ is $1-L C$ at $x$; the local homotopy type of $S^{1}$ at a vertex $v$ is the homotopy type of $S^{0} * M^{3}$, which is also simply connected. Thus $S^{1}$ is a locally nicely imbedded 1 -sphere in $S^{5}$, and hence is flat, [1, Theorem 4.2]. Thus $L * K$ is a locally flat, noncombinatorial triangulation of $S^{5}$.

\section{REFERENCES}

1. J. L. Bryant and C. L. Seebeck, Locally nice embeddings of polyhedra, Quart. J. Math. Oxford Ser. (2) 19 (1968), 257-74.

2. L. C. Glaser, On the double suspension of certain homotopy 3-spheres, Ann. of Math. (2) 85 (1967), 494-507. MR 36 \#886.

University OF GeORgIA 